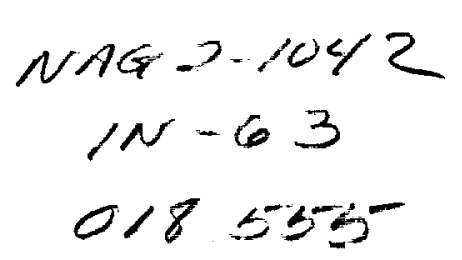

\title{
Approximated Stable Inversion for Nonlinear Systems with Nonhyperbolic Internal Dynamics *
}

\author{
Santosh Devasia ${ }^{\dagger}$ \\ Dept. of Mechanical Engineering \\ U. of Utah, SLC, UT 84112, USA
}

Abstract - A technique to achieve output tracking for nonminimum phase nonlinear systems with nonhyperbolic internal dynamics is presented. The present paper integrates stable inversion techniques (that achieve exact-tracking) with approximation techniques (that modify the internal dynamics) to circumvent the nonhyperbolicity of the internal dynamics - this nonhyperbolicity is an obstruction to applying presently available stable inversion techniques. The theory is developed for nonlinear systems and the method is applied to a two-cart with inverted-pendulum example.

\footnotetext{
- Work Supported by NASA Ames Research Center Grant NAG-2-1042

te-mail : santosh@eng utah.edu Tel: (801) 5814613
} 


\section{Introduction}

Precision output tracking controllers are needed to meet increasingly stringent performance requirements in applications like flexible structures, aircraft guidance, robotics, and manufacturing systems. While exact tracking of minimum phase systems is relatively easy to achieve through approaches like input-output linearization [1], output tracking of nonminimum phase systems tends to be more challenging due to fundamental performance limitations on transient tracking performance [2]. This poor transient performance has been mitigated by using pre-actuation in the stable-inversion based approaches $[3,4]$. However, the preactuation time (during which most of the preactuation control effort is required) depends on the unstable poles of the linearized internal dynamics - the preactuation time increases as the unstable poles approach the imaginary axis. In the limiting case, with the poles on the imaginary axis (nonhyperbolic internal dynamics), presently available inversion-based techniques are not applicable and the effective preactuation time tends to become infinite. The present work extends previous results for linear systems in [5] to output tracking of nonlinear nonminimum phase systems, which have nonhyperbolic internal dynamics.

Output tracking has a long history marked by the development of regulator theory for linear systems by Francis and Wonham [6] and the generalization to the nonlinear case by Byrnes and Isidori [7]. These approaches asymptotically track an output from a class of exosystem-generated outputs. Further, extensions to the Byrnes-Isidori regulator have been described in [8]. The main problem with the application of these techniques to the output tracking of nonlinear systems is computational. While the linear regulator is designed by solving a manageable set of linear equations, the nonlinear regulator design requires the nontrivial solution of a first order partial differential algebraic equation $[9,10]$. In contrast, inversion-based approaches avoid this computational difficulty and trade it to solve the exact tracking problem for a single desired output trajectory rather than solve the asymptotic tracking for a class of outputs. Another problem with the regulator approach is that the exosystem states are often switched to describe the desired output - this leads to transient tracking-errors after the switching instants. Such switching caused transient errors can be avoided by using inversion-based approaches to output tracking $[3,11]$. Thus, it is advantageous to use inversion-based output tracking when precision tracking of a particular output trajectory is required.

Inversion, which is key to our approach, was restricted to causal inverses of minimum phase systems in the early works by Silverman and by Hirschorn (e.g., $[12,13])$ because these approaches lead to unbounded inverses in the nonminimum phase case. Di Benedetto and Lucibello [14] considered the inversion of time varying nonminimum phase systems with a choice of the system's initial conditions. Rather than choose initial conditions, preactuation was used by noncausal stable inversion techniques developed in $[3,4,15]$. Such noncausal inverses, which require preactuation, have been successfully applied to the output tracking of flexible structures $[16,17]$, and aircraft and air traffic control $[4,18]$. There is, however, a fundamental limitation to the presently available inversion techniques - they are only applicable if the internal dynamics is hyperbolic, and inversion-based output tracking has been a challenge for systems with nonhyperbolic internal dynamics.

Although Huang [8] has proposed some sufficient conditions for developing a regulator for systems with nonhyperbolic internal dynamics, the regulator design remains computationally difficult. There are also several approximation based output tracking techniques, where the central philosophy is to replace the internal dynamics with a dynamics that provides satisfactory behavior, and then to develop the controller based on the altered system [19, 20,21]. The techniques most relevant to this paper are developed by Gurumoorthy and Sanders [19], and by Gopalswamy and Hedrick [21] - output redefinition (or modification) is used in these to approximate the unstable internal dynamics with a stable system. Such approximation based approaches are integrated, in this paper, with stable-inversion based techniques to achieve inversion of linear systems with nonhyperbolic internal dynamics in [5]. However, the present technique does not use output modification to stabilize the internal dynamics, rather output modification is only used to remove the nonhyperbolicity. Additionally, for near nonhyperbolic systems, the present approach allows a tradeoff between the precision-tracking and the amount of pre-actuation-time that is needed in the control effort. This tradeoff between stable-inversion and approximation of the unacceptable internal-dynamics has been studied for helicopter-hover control in [5]. The present work extends the results for linear-systems in [5] to nonlinear systems.

We begin with a background on the stable-inversion based output tracking technique, and computational issues are presented in Section 3. The technique is applied to an example in Section 4, and conclusions are 
in Section 5.

\section{The Stable Inversion problem}

\subsection{Inversion-Based Output Tracking Scheme}

Here we describe how the inversion approach is used to develop output tracking controllers. Consider a system described by

$$
\begin{aligned}
& \dot{x}(t)=f[x(t)]+g[x(t)] u(t) \\
& y(t)=h[x(t)]
\end{aligned}
$$

where $y(t)=\left[y_{1}(t), y_{2}(t), \ldots, y_{p}(t)\right]^{T}$ is the output, with the same number of inputs and outputs, i.e., $u(t), y(t) \in \Re^{p}$, and $x(t) \in \Re^{n}$ is the state. The functions $f(\cdot), g(\cdot)$ and $h(\cdot)$ are assumed to be sufficiently smooth with $f(0)=0$ (i.e., $x=0$ is an equilibrium point) and $h(0)=0$.

Let $y_{d}(\cdot)$ be the desired output trajectory to be tracked. In the inversion-based approach we, first, find a bounded, nominal input-state trajectory, $\left[u_{f f}(\cdot), x_{r e f}(\cdot)\right]$ that satisfies the system equations (1) and yields the desired output exactly, i.e.,

$$
\left.\begin{array}{rl}
\dot{x}_{\text {ref }}(t) & =f\left[x_{\text {ref }}(t)\right]+g\left[x_{\text {ref }}(t)\right] u_{f f}(t) \\
y_{d}(t) & =h\left[x_{\text {ref }}(t)\right]
\end{array}\right\} \quad \forall t \in(-\infty, \infty)
$$

and, second, we use the exact-output yielding input trajectory, $u_{f f}(\cdot)$, as feedforward and the system is stabilized by using feedback. It is noted that in this output tracking scheme, the feedforward input $u_{f f}(\cdot)$ is computed off-line.

In the absence of modeling errors, initial condition errors, and external disturbances, exact-tracking can be achieved by the use of feedforward alone. If the system (1) is stable (or stabilized with feedback before the inversion is applied) then standard Lyapunov arguments [22] can be used to show that, with the use of feedforward, $x(t) \rightarrow x_{\text {ref }}(t)$ and $y(t) \rightarrow y_{d}(t)$ as $t \rightarrow \infty$ and output tracking is achieved [3,15]. The feedback scheme can also include integrals of the tracking error [22] to obtain zero steady-state errors (see Figure 1). While stabilization of the system can be achieved through standard techniques, the main challenge is to find the inverse input-state trajectory $\left[u_{f f}(\cdot), x_{r e f}(\cdot)\right]$ - especially for systems with nonminimum phase dynamics. This paper addresses the issue of generating the inverse input-state trajectory for a system and can be used in conjunction with any feedback stabilization scheme.

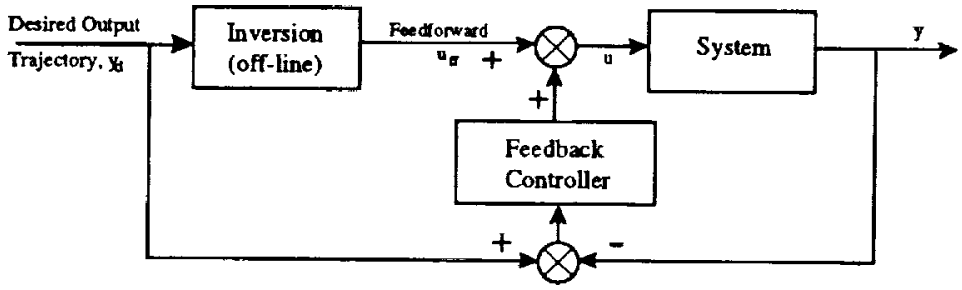

Figure 1. The Control Scheme

\subsection{The Internal Dynamics}

In this subsection, it is shown that finding the inverse input-state trajectory is equivalent to finding bounded solutions to the system's internal dynamics.

Assumption 1 System (1) has a well defined vector relative degree, $r:=\left[r_{1}, r_{2}, \ldots, r_{p}\right]$.

The well-defined vector relative degree assumption enables the system equations to be rewritten, through a co-ordinate transformation

$$
x(t)=\dot{T}(x(t))\left[\begin{array}{l}
\zeta(t) \\
\eta(t)
\end{array}\right]=T(\zeta(t), \eta(t))\left[\begin{array}{l}
\zeta(t) \\
\eta(t)
\end{array}\right]
$$


in the following form $[1,21]$

$$
\begin{aligned}
& \dot{\zeta}_{1}(t)=A_{\zeta_{1}} \zeta_{1}(t)+A_{\zeta_{2}} \zeta_{2}(t) \\
& \dot{\zeta}_{2}(t)=s_{1}[\zeta(t), \eta(t)]+s_{2}[\zeta(t), \eta(t)] u(t) \\
& \dot{\eta}(t)=s_{3}[\zeta(t), \eta(t)]+s_{4}[\zeta(t), \eta(t)] u(t)
\end{aligned}
$$

where $\zeta$ represents the output along with its time derivatives, $\zeta$ is partitioned as $\left[\zeta_{1}(t) \vdots \zeta_{2}(t)\right]^{T}$ and is given by

$$
\left[y_{1} \frac{d y_{1}}{d t} \frac{d^{2} y_{1}}{d t^{2}} \ldots \frac{d^{r_{1}-2} y_{1}}{d t^{r_{1}-2}} y_{2} \ldots \frac{d^{r_{3}-2}}{d t^{r_{2}-2}} y_{2} \ldots \frac{d^{r_{p}-2}}{d t^{r_{p}-2}} y_{p} \quad \vdots \frac{d^{r_{1}-1}}{d t^{r_{1}-1}} y_{1} \frac{d^{r_{2}-1}}{d t^{r_{2}-1}} y_{2} \ldots \frac{d^{r_{p}-1}}{d t^{r_{p}-1}} y_{p} .\right]^{T}
$$

Further $A_{\zeta_{1}}$ is a block-diagonal matrix, $A_{\zeta_{1}}=\operatorname{diag}\left[A_{1}, A_{2}, A_{3}, \ldots, A_{p}\right]$, with each block consisting of zeros except for ones on the super-diagonal $\left(A_{k}\right.$ is a $\left(r_{k}-1\right) \times\left(r_{k}-1\right)$ matrix for each $\left.1 \leq k \leq p\right)$. $A_{\zeta_{2}}$ is a matrix whose elements are zeros except for elements on the $\left(r_{1}+r_{2}+\ldots+r_{k}-k\right)$-th row and $k$-th column, which are ones (for all $1 \leq k \leq p$ ). Further, from Assumption (1), $s_{2}(\cdot, \cdot)$ is invertible in a neighborhood of the origin, and since the origin is assumed to be an equilibrium point, we also have $s_{1}(0,0)=0, s_{3}(0,0)=0$.

Note that the desired $\zeta(\cdot)$ is known when the desired output trajectory $y_{d}(\cdot)$ and its time derivatives are specified. This desired $\zeta(\cdot)$ is defined as $\zeta_{d}(\cdot)$. If exact output tracking is achieved (i.e., if $\zeta(t)=\zeta_{d}(t)$ ) then the control law for maintaining exact tracking can be written as, from equation (4),

$$
u(t)=\left[s_{2}\left[\zeta_{d}(t), \eta(t)\right]\right]^{-1}\left[\dot{\zeta}_{2, d}(t)-s_{1}\left[\zeta_{d}(t), \eta(t)\right]\right]
$$

which results in state-equations of the form

$$
\begin{aligned}
\dot{\zeta}(t) & =\dot{\zeta}_{d}(t) \\
\dot{\eta}(t) & =s_{3}[\zeta(t), \eta(t)]+s_{4}[\zeta(t), \eta(t)] u(t) \\
& =s_{3}[\zeta(t), \eta(t)]+s_{4}[\zeta(t), \eta(t)]\left[s_{2}\left(\zeta_{d}(t), \eta(t)\right)\right]^{-1}\left[\dot{\zeta}_{2, d}(t)-s_{1}\left[\zeta_{d}(t), \eta(t)\right]\right]
\end{aligned}
$$

This is the inverse system and equation (8) is referred to as the internal dynamics. Solving the internal dynamics is key to finding the inverse input state trajectories. If a bounded solution, $\eta_{d}(\cdot)$, to the internal dynamics (8) can be found, then the feedforward input can be found through equation (6) as

$$
u_{\rho \delta}(t)=\left[s_{2}\left(\zeta_{d}(t), \eta_{d}(t)\right)\right]^{-1}\left[\dot{\zeta}_{2, d}(t)-s_{1}\left(\zeta_{d}(t), \eta_{d}(t)\right)\right]
$$

and the reference state trajectory can be found as

$$
x_{r e f}(t)=T\left[\zeta_{d}(t), \eta_{d}(t)\right]\left[\begin{array}{l}
\zeta_{d}(t) \\
\eta_{d}(t)
\end{array}\right]
$$

Thus, finding a bounded solution, $\eta_{d}(\cdot)$, to the internal dynamics (Equation 8 ) is key to finding the inverse (bounded) input-state trajectories (Equations 9 and 10) which are needed to implement the inversion-based output-tracking scheme shown in Figure 1.

\subsection{Modified Internal Dynamics}

Standard inversion schemes $[12,13]$ that integrate (forward in time) the internal dynamics $(8)$ lead to unbounded solutions if the origin of the internal dynamics is unstable (nonminimum phase systems). Noncausal inversion (e.g., [3]) leads to bounded but noncausal solution of the internal dynamics. While significant improvement in output tracking performance is possible, such stable-inversion techniques are not applicable to 
systems with nonhyperbolic internal dynamics. In this subsection a compromise between stable inversion and approximation-based inversion schemes is proposed. The key is to modify the internal dynamics by giving up exact output tracking - enough to remove the nonhyperbolicity, and then to apply stable-inversion. The difference between the proposed technique and other approximate techniques is that the internal dynamics is perturbed only to remove the nonhyperbolicity, and not to stabilize the internal dynamics as in other approximation schemes $[19,21]$.

To modify the inverse system an extra term, $v(t)$, is added to the control law (6) as follows

$$
u(t)=\left[s_{2}[\zeta(t), \eta(t)]\right]^{-1}\left[\dot{\zeta}_{2, d}(t)-s_{1}[\zeta(t), \eta(t)]+v(t)\right]
$$

Note that the modified input does not attempt to achieve exact tracking of the desired output (i.e., $\zeta(\cdot)=\zeta_{d}(\cdot)$ is not required) Therefore, the modification, $v(t)$, of the control law can be used to modify the internal dynamics. With this control law, the system equation (4) becomes

$$
\begin{aligned}
\dot{\zeta}_{1}(t) & =A_{\zeta_{1}} \zeta_{1}(t)+A_{\zeta_{2}} \zeta_{2}(t) \\
\dot{\zeta}_{2}(t) & =\dot{\zeta}_{2, d}(t)+v(t) \\
\dot{\eta}(t) & =s_{3}[\zeta(t), \eta(t)]+s_{4}[\zeta(t), \eta(t)]\left[s_{2}[\zeta(t), \eta(t)]\right]^{-1}\left[\dot{\zeta}_{2, d}(t)-s_{1}[\zeta(t), \eta(t)]+v(t)\right]
\end{aligned}
$$

For ease in notation, we define the tracking error, $e_{\zeta}(t)$, as

$$
e_{\zeta}(t):=\left[\begin{array}{l}
e_{\zeta_{1}}(t) \\
e_{\zeta_{2}}(t)
\end{array}\right]:=\left[\begin{array}{l}
\zeta_{1}(t)-\zeta_{1, d}(t) \\
\zeta_{2}(t)-\zeta_{2, d}(t)
\end{array}\right]
$$

and rewrite Equation (12) as

$$
\begin{aligned}
\dot{e}_{\zeta_{1}}(t)= & A_{\zeta_{1}} e_{\zeta_{1}}(t)+A_{\zeta_{2}} e_{\zeta_{2}}(t) \\
\dot{e}_{\zeta_{2}}(t)= & v(t) \\
\dot{\eta}(t)= & s_{3}\left[e_{\zeta}(t)+\zeta_{d}(t), \eta(t)\right]+ \\
& s_{4}\left[e_{\zeta}(t)+\zeta_{d}(t), \eta(t)\right]\left[s_{2}\left[e_{\zeta}(t)+\zeta_{d}(t), \eta(t)\right]\right]^{-1}\left[\dot{\zeta}_{2, d}(t)-s_{1}\left[e_{\zeta}(t)+\zeta_{d}(t), \eta(t)\right]+v(t)\right] \\
:= & \hat{s}\left[e_{\zeta}(t), \eta(t), Y_{d}(t), v(t)\right]
\end{aligned}
$$

where

$$
Y_{d}(t):=\left[\begin{array}{c}
\zeta_{d}(t) \\
\dot{\zeta}_{2, d}(t)
\end{array}\right]
$$

If $v(t)=0$ then Equation (13) represents the inverse-system, which has to be solved to find the exacttracking inverse input-state trajectories. However, the internal dynamics part of equation (13),

$$
\dot{\eta}(t)=\hat{s}\left[e_{\zeta}(t), \eta(t), Y_{d}(t), 0\right],
$$

is nonhyperbolic and may not have a bounded solution (see the Example in Section 4) - thus the inversion problem may not have a bounded solution. Even when bounded solutions exist for the nonhyperbolic internal dynamics, currently available computational techniques for stable-inversion cannot be directly used since they require hyperbolicity of the internal dynamics $[3,4]$. Our approach is to appropriately choose $v(\cdot)$ to remove the nonhyperbolicity of the inverse system (13), which includes the internal dynamics. We begin with the following assumption.

Assumption 2 System (1) is controllable in the first approximation [1].

The above assumption implies that the modified inverse system is also controllable in the first approximation since the difference between the original system (1) and the modified internal dynamics (13) is only a 
co-ordinate transformation and a static state-feedback (see [21] for a similar argument used for output redefinition). Next, a feedback of the form

$$
v(t)=F\left[\begin{array}{c}
e_{\zeta}(t) \\
\eta(t)
\end{array}\right]
$$

is chosen such that the modified inverse system is hyperbolic - i.e., all poles on the imaginary axis are moved. Note that this change to a hyperbolic system can be achieved through an arbitrarily small $F$ since nonhyperbolicity is not a structurally stable property. With this control law Equation (13) becomes

$$
\begin{aligned}
\dot{e}_{\zeta_{1}}(t) & =A_{\zeta_{1}} e_{\zeta_{1}}(t)+A_{\zeta_{2}} e_{\zeta_{2}}(t) \\
\dot{e}_{\zeta_{2}}(t) & =F\left[\begin{array}{c}
e_{\zeta}(t) \\
\eta(t)
\end{array}\right] \\
\dot{\eta}(t) & =\hat{s}\left(e_{\zeta}(t), \eta(t), Y_{d}(t), F\left[\begin{array}{c}
e_{\zeta}(t) \\
\eta(t)
\end{array}\right]\right)
\end{aligned}
$$

which is re-written in a simplified form as

$$
\left[\begin{array}{c}
\dot{e}_{\zeta}(t) \\
\dot{\eta}(t)
\end{array}\right]=s\left(e_{\zeta}(t), \eta(t), Y_{d}(t)\right)
$$

Next, stable inversion of the modified inverse system (16) is carried out [3].

\section{Computation of the Inverse}

This section discusses the computation of the inverse for the hyperbolic, modified, inverse-systern. First, an iterative algorithm is presented - one of the steps involves finding bounded solutions to a linear (unstable) system. Second, the explicit technique to find bounded solutions is presented, and the amount of preactuation required for implementing the inversion-based controller is discussed. We begin with the algorithm to find bounded solutions to the modified internal dynamics [3].

\subsection{Iterative Algorithm to find Inverse}

\section{- Step 1}

Rewrite the modified inverse (16) as

$$
\frac{d}{d t}\left[\begin{array}{c}
e_{\zeta}(t) \\
\eta(t)
\end{array}\right]=S\left[\begin{array}{c}
e_{\zeta}(t) \\
\eta(t)
\end{array}\right]+\left\{s\left(e_{\zeta}(t), \eta(t), Y_{d}(t)\right)-S\left[\begin{array}{c}
e_{\zeta}(t) \\
\eta(t)
\end{array}\right]\right\}
$$

where the first term on the right hand side (r.h.s.) is the linearization of $s(\cdot, \cdot, \cdot)$ with respect to the first two variables, $e_{\zeta}(\cdot)$ and $\eta(\cdot)$, and the second term on the r.h.s. represents a perturbation. This motivates the following iterative solution of the internal dynamics.

- Step 2

We begin with $\left[e_{\zeta}(t) \eta(t)\right]_{0}^{T}=0$ and then, at at each iteration in the following scheme, the bounded solution of the following linearized system-equation is found.

$$
\frac{d}{d t}\left[\begin{array}{c}
e_{\zeta}(t) \\
\eta(t)
\end{array}\right]_{N+1}=S\left[\begin{array}{c}
e_{\zeta}(t) \\
\eta(t)
\end{array}\right]_{N+1}+\left\{s\left(e_{(\eta, N)}(t), \eta_{N}(t), Y_{d}(t)\right)-S\left[\begin{array}{c}
e_{\zeta}(t) \\
\eta(t)
\end{array}\right]_{N}\right\}
$$

where

and $N=1,2,3 \ldots$.

$$
\left[\begin{array}{c}
e_{\zeta}(t) \\
\eta(t)
\end{array}\right]_{N+1}:=\left[\begin{array}{c}
e_{\zeta, N+1}(t) \\
\eta_{N+1}(t)
\end{array}\right]
$$




\subsection{Bounded Solution to Unstable Linear System}

In the above iterations, solving equation (18) implies finding a bounded solution to a linear, hyperbolic (but potentially unstable) system - this is described next.

We begin by decoupling the linear system (18) into stable $\left(z_{s}\right)$ and unstable $\left(z_{u}\right)$ subsystems. Since the modified internal dynamics is hyperbolic, there exits a decoupling transformation $U$ such that equation (18) can be re-written as

$$
\begin{aligned}
& \dot{z}_{(s, N+1)}(t)=S_{s} z_{(s, N+1)}(t)+G_{s} Y_{N}(t) \\
& \dot{z}_{(u, N+1)}(t)=S_{u} z_{(u, N+1)}(t)+G_{u} Y_{N}(t)
\end{aligned}
$$

where

$$
z_{N+1}(t):=\left[\begin{array}{c}
z_{(s, N+1)}(t) \\
z_{(u, N+1)}(t)
\end{array}\right]:=U\left[\begin{array}{c}
e_{(\eta, N+1)}(t) \\
\eta_{N+1}(t)
\end{array}\right]
$$

and

$$
Y_{N}(t):=\left\{s\left(e_{(\eta, N)}(t), \eta_{N}(t), Y_{d}(t)\right)-S\left[\begin{array}{c}
e_{\zeta}(t) \\
\eta(t)
\end{array}\right]_{N}\right\}
$$

An approach to find bounded solutions is to enforce the boundary conditions that $z_{(s, N+1)}(-\infty)=0$ and $z_{(u, N+1)}(\infty)=0$. This leads to unique bounded solutions by flowing the stable subsystem forward in time and flowing the unstable system backward in time - this yields (see [15] for computational issues)

$$
\begin{array}{ll}
z_{(s, N+1)}(t)=\int_{-\infty}^{t} e^{S_{u}(t-\tau)} G_{s} Y_{N}(\tau) d \tau & \forall t \in(-\infty, \infty), \\
z_{(u, N+1)}(t)=\int_{\infty}^{t} e^{-S_{u}(\tau-t)} G_{u} Y_{N}(\tau) d \tau & \forall t \in(-\infty, \infty) .
\end{array}
$$

Next, a change of co-ordinates yields the bounded solution to (18) as

$$
\left[\begin{array}{c}
e_{\zeta}(t) \\
\eta(t)
\end{array}\right]_{N+1}=U^{-1}\left[\begin{array}{c}
z_{(s, N+1)}(t) \\
z_{(u, N+1)}(t)
\end{array}\right]_{N+1} .
$$

\subsection{Convergence of Iterative Algorithm}

The following Theorem states that, as $N \rightarrow \infty$, the solutions of the above iterative algorithm converge to a bounded solution of the modified inverse system (16).

Theorem Let the hyperbolic, modified, internal dynamics $s(\cdot, \cdot, \cdot)$ and its linearization $S$ satisfy the following Lipschitz like condition on a neighborhood of the origin, $(0,0,0)$,

$$
\begin{gathered}
\left\|\left\{s\left(e_{1}, \eta_{1}, Y_{1}\right)-S\left[\begin{array}{c}
e_{1} \\
\eta_{1}
\end{array}\right]\right\}-\left\{s\left(e_{2}, \eta_{2}, Y_{2}\right)-S\left[\begin{array}{c}
e_{2} \\
\eta_{2}
\end{array}\right]\right\}\right\|_{\infty} \\
\leq K_{1}\left\|\left[\begin{array}{c}
e_{1} \\
\eta_{1}
\end{array}\right]-\left[\begin{array}{c}
e_{2} \\
\eta_{2}
\end{array}\right]\right\|_{\infty}+K_{2}\left\|Y_{1}-Y_{2}\right\|_{\infty}
\end{gathered}
$$

Then, provided $K_{1}, K_{2}$ and $\left\|Y_{d}(\cdot)\right\|_{\infty}$ are small, the iteration scheme converges to a solution of the modified inverse system (16), i.e,

$$
\left[\begin{array}{c}
e_{\zeta} \\
\eta
\end{array}\right]_{N} \rightarrow\left[\begin{array}{c}
e_{(\zeta, d)} \\
\eta_{d}
\end{array}\right]
$$

as $N \rightarrow \infty$, and $\left[\begin{array}{c}e_{(\zeta, d)} \\ \eta_{d}\end{array}\right]$ satisfies the modified inverse system.

Proof

See $[3,4]$.

Thus, stable inversion technique is applied after removing the nonhyperbolicity in the internal dynamics. 
Remark 1 The conditions in the theorem are sufficient conditions for finding bounded solutions to the internal dynamics. They are not, however, necessary for the existence of bounded solutions to the internal dynamics - bounded solutions can exist even when the internal dynamics are non-hyperbolic. Further, $S$ in the above theorem can also be chosen to be different from the linearization of the nonlinearity $s$, however, additional conditions have to be imposed (see, [3], Condition 1 in Definition 5).

This completes the inversion-technique for nonminimum phase systems with nonhyperbolic internal dynamics. To summarize, the reference state trajectory is found as (from Equation 10)

$$
x_{r e f}(t)=T\left[\hat{\zeta}_{d}(t), \eta_{d}(t)\right]\left[\begin{array}{c}
\hat{\zeta}_{d}(t) \\
\eta_{d}(t)
\end{array}\right]
$$

where

$$
\hat{\zeta}_{d}(t):=\zeta_{d}(t)+e_{(\zeta, d)}(t)
$$

and the feedforward input, $u_{f f}(\cdot)$, is found from equation (11) and equation (14) as

$$
u_{f f}(t)=\left[s_{2}\left[\tilde{\zeta}_{d}(t), \eta_{d}(t)\right]\right]^{-1}\left[\dot{\zeta}_{2, d}(t)-s_{1}\left[\hat{\zeta}_{d}(t), \eta(t)\right]+F\left[\begin{array}{c}
e_{(\zeta, d)}(t) \\
\eta_{d}(t)
\end{array}\right]\right]
$$

The inverse input-state trajecotry, $\left[u_{f f}(\cdot), x_{r e f}(\cdot)\right]$, is then used with feedback stabilization to obtain output tracking [3].

\subsection{Error Analysis}

The following Lemma establishes an upper bound on the tracking error, $e_{(\zeta, d)}$, for a given choice of feedback, $F$, that removes the nonhyperbolicity of the inverse system (15).

Lemma 1 Let

$$
\phi(t)=U^{-1}\left[\begin{array}{cc}
I(t) e^{5, t} & 0 \\
0 & -I(-t) e^{-S_{u} t}
\end{array}\right]
$$

where $I(\cdot)$ is the unit step function, $S$ is defined from equation (17) as

$$
S=\left[\begin{array}{ccc}
A_{\zeta_{1}} & A_{\zeta_{2}} & 0 \\
& F & \\
& \hat{S} &
\end{array}\right]
$$

where $\hat{S}$ is the linearization of $\hat{s}(\cdot, \cdot, \cdot)$ with respect to the variables, $e_{\zeta}(\cdot)$ and $\eta(\cdot)$ (see equation (15)), and $S_{s}$ and $S_{u}$ represent the decomposition of linearized inverse system into stable and unstable parts by the co-ordinate transformation, $U$, as discussed in Subsection 3.2. Further, let

$$
\|\phi(\cdot)\|_{1}=n \max _{i, j}\left\|\phi_{i, j}(\cdot)\right\|_{1} .
$$

Then the tracking error for a particular choice of $F$ can be bounded as

$$
\left\|e_{\zeta_{d}}(t)\right\|_{\infty} \leq \frac{\|\phi(\cdot)\|_{1} K_{2}\left\|Y_{d}(\cdot)\right\|_{\infty}}{1-K_{1}\|\phi(\cdot)\|_{1}}
$$

Proof This follows from Theorem 3.6 in [23].

\subsection{Preactuation Time}

Stable-inversion techniques overcome fundamental limitations on transient tracking performance of nonminimum phase systems [2] by using preactuation. However, the preactuation time (i.e., when most of the preactuation effort is required) tends to be unacceptably large if the unstable poles of the linearized, modified internal dynamics are close to the imaginary axis - this dependence is established in the following Lemma. 


\section{Lemma 2}

Let

- all the unstable poles of linearized, modified inverse system (eigenvalues of $S_{u}$ ) lie to the right, in the complex plane, of the line $\operatorname{Real}(s)=\alpha$ for some positive $\alpha$, and

- the support of $Y_{d}(\cdot)$ lie in $\left[t_{0}, \infty\right)$ for some $t_{0}$.

Then there exists a positive scalar, $M$, such that the bounded solution to the internal dynamics (defined by the Theorem) satisfies

$$
\left\|\underset{\eta_{d}(t)}{e_{(\zeta, d)}(t)}\right\|_{\infty}<M e^{\frac{\sigma}{3}\left(t-t_{0}\right)}
$$

for all time, $t$, before the start of the maneuver at $t_{0}$.

Proof Since the maneuver starts at $t_{0}$, any solution to the modified internal dynamics (16) satisfies the autonomous equation,

$$
\left[\begin{array}{c}
\dot{e}_{\zeta}(t) \\
\dot{\eta}(t)
\end{array}\right]=s\left(e_{\zeta}(t), \eta(t), 0\right)
$$

for all $t<t_{0}$. Further, any bounded solution to the autonomous equation must lie on the unstable manifold before the start of the maneuver. The rate of convergence to zero as time tends to $-\infty$, of solutions that lie on the unstable manifold of the nonlinear modified inverse system, directly depends on the location of poles of the linearized dynamics. In particular, the rate of convergence depends on the unstable poles of the linearization (which are the eigenvalues of $S_{u}$ ). The existence of a positive scalar $M$ that satisfies the statement of the lemma follows from the saddle-point property (see [24] Theorem 6.1, and [25] Theorem 19.9).

The present technique also provides a way to reduce the preactuation time for systems with near nonhyperbolic internal dynamics. The Lemma states that the desired state-trajectory exponentially tends to zero as we go back in time before the start of the maneuver at $t_{0}$. Then the continuity of the input with respect to the state (smoothness of $f, g$ and $h$, and the well defined relative degree assumption) implies that the preactuation input also tends to zero exponentially. The rate at which the preactuation becomes zero can be increased by moving the unstable poles of the linearized, modified inverse system (i.e., the eigenvalues of $S_{u}$ ) away from the imaginary axis - by appropriately choosing $F$ in equation (14). This reduction in preactuation-effort is obtained at the expense of exact output tracking (see [5] for a linear example).

\section{Example}

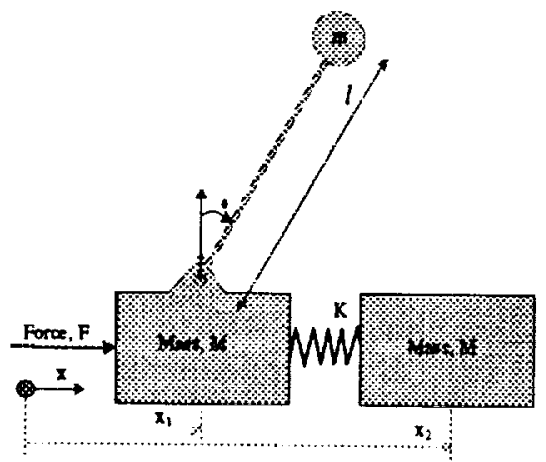

Figure 2. Example: Two Carts and Inverted Pendulum

Here, the inversion-technique is applied to an example two-cart and pendulum system shown in Figure 2. The inverted pendulum on a cart has been well studied in literature (see, for example, [19]), and has nonminimum phase dynamics - the internal dynamics is hyperbolic. An extra cart is added here, which introduces nonhyperbolicity in the internal dynamics. The input to the system is the applied force, $F(t)$, 
and the output is the position, $x_{1}(t)$, of the cart carrying the inverted pendulum (see Figure 2). The equation of motion for the system can be obtained as

$$
\begin{aligned}
(M+m) \ddot{x}_{1}(t)+m l \cos \theta(t) \ddot{\theta}(t) & =F(t)+m l \dot{\theta}^{2}(t) \sin \theta(t)-K\left(x_{1}(t)-x_{2}(t)\right) \\
m l \cos \theta(t) \ddot{x}_{1}(t)+m l^{2} \ddot{\theta}(t) & =m g l \sin \theta(t) \\
M \ddot{x}_{2}(t) & =-K\left(x_{2}(t)-x_{1}(t)\right)
\end{aligned}
$$

which can be rewritten in state-space form as

$$
\begin{aligned}
\dot{\zeta}_{1}(t)= & \zeta_{2}(t) \\
\dot{\zeta}_{2}(t)= & \frac{1}{\gamma\left(\eta_{3}\right)}\left[F(t) / m+l \eta_{4}(t)^{2} \sin \eta_{3}(t)-K / m\left(\zeta_{1}(t)-\eta_{1}(t)\right)-g \cos \eta_{3}(t) \sin \eta_{3}(t)\right] \\
\dot{\eta}_{1}(t)= & \eta_{2}(t) \\
\dot{\eta}_{2}(t)= & (K / M)\left(\zeta_{1}(t)-\eta_{1}(t)\right) \\
\dot{\eta}_{3}(t)= & \eta_{4}(t) \\
\dot{\eta}_{4}(t)= & \frac{1}{\gamma\left(\eta_{3}\right)}\left[-\eta_{4}(t)^{2} \sin \eta_{3}(t) \cos \eta_{3}(t)\right. \\
& \left.\quad+\frac{1}{m}\left\{(M+m) g \sin \eta_{3}(t)-F(t) \cos \eta_{3}(t)+K\left(\zeta_{1}(t)-\eta_{1}(t)\right) \cos \eta_{3}(t)\right\}\right]
\end{aligned}
$$

where $\zeta_{1}:=x_{1}, \quad \zeta_{2}:=\dot{x}_{1}, \quad \eta_{1}:=x_{2}, \quad \eta_{2}:=x_{2}, \quad \eta_{3}:=\theta$, and $\eta_{4}:=\dot{\theta}$, and $\gamma\left(\eta_{3}(t)\right):=M / m+\left[\sin \eta_{3}(t)\right]^{2}$ Given the desired output trajectory profile, the input that maintains exact tracking (i.e., which maintains $\left.\zeta(t)=\zeta_{d}(t)\right)$ is obtained from equation $(26)$ as

$$
F(t)=m \gamma\left(\eta_{3}\right) \ddot{x}_{1, d}(t)-m l\left[\eta_{4}(t)\right]^{2} \sin \eta_{3}(t)+K\left[\zeta_{1}(t)-\eta_{1}(t)\right]+m g\left[\cos \eta_{3}(t)\right] \sin \eta_{3}(t)
$$

With this exact tracking control law, the linearized internal dynamics (represented by $\eta$ ), is given by

$$
\frac{d}{d t}\left[\begin{array}{l}
\eta_{1}(t) \\
\eta_{2}(t) \\
\eta_{3}(t) \\
\eta_{4}(t)
\end{array}\right]=\left[\begin{array}{cccc}
0 & 1 & 0 & 0 \\
-K / M & 0 & 0 & 0 \\
0 & 0 & 0 & 1 \\
0 & 0 & g / l & 0
\end{array}\right]\left[\begin{array}{l}
\eta_{1}(t) \\
\eta_{2}(t) \\
\eta_{3}(t) \\
\eta_{4}(t)
\end{array}\right]+\left[\begin{array}{cc}
0 & 0 \\
K / M & 0 \\
0 & 0 \\
0 & -1 / l
\end{array}\right]\left[\begin{array}{l}
x_{1, d}(t) \\
\ddot{x}_{1, d}(t)
\end{array}\right]
$$

Note that the linearization has two poles at $\pm \sqrt{-K / M}$ on the imaginary axis (in the complex plane) which correspond to the cart dynamics $\left(x_{2}, \dot{x}_{2}\right)$ with $x_{1}$ fixed; i.e., a spring-mass system. The other two poles are at $\pm \sqrt{g / l}$ which correspond to the linearization of the inverted pendulum dynamics $(\theta, \dot{\theta})$. The two poles on the imaginary axis lead to nonhyperbolic behavior and stable-inversion techniques fail - this corresponds to the case which requires infinite pre-actuation.

Remark 2 Note that the nonhyperbolic spring-mass system will not have a bounded solution if the desired output excites the spring-mass system at its resonance frequency. Thus the nonhyperbolicity of the internal dynamics can imply that exact-tracking cannot be achieved for some desired-output trajectories.

By modifying the desired trajectory, the internal dynamics can be approximated by a hyperbolic system, for which existing stable-inversion techniques are applicable. This modification is achieved by adding an extra term $v(\cdot)$ to the control as follows

$$
\begin{aligned}
u_{f f}(t)=F(t)= & m \gamma\left(\eta_{3}\right)\left[\ddot{x}_{1, d}(t)+v(t)\right]-m l\left[\eta_{4}(t)\right]^{2} \sin \eta_{3}(t) \\
& +K\left[\zeta_{1}(t)-\eta_{1}(t)\right]+m g\left[\cos \eta_{3}(t)\right] \sin \eta_{3}(t)
\end{aligned}
$$

The modified inverse system becomes

$$
\begin{aligned}
\dot{e}_{\zeta_{3}}(t) & =e_{\zeta_{3}}(t) \\
\dot{e}_{\zeta_{3}}(t) & =v(t) \\
\dot{\eta}_{1}(t) & =\eta_{2}(t) \\
\dot{\eta}_{2}(t) & =-K / M \eta_{1}+K / M\left[x_{1, d}(t)+e_{\zeta_{1}}(t)\right] \\
\dot{\eta}_{3}(t) & =\eta_{4}(t) \\
\dot{\eta}_{4}(t) & =g / l \sin \eta_{3}(t)-1 / l \cos \eta_{3}(t)\left[\ddot{x}_{1, d}(t)+v(t)\right]
\end{aligned}
$$


where

$$
e_{\zeta}(t):=\left[\begin{array}{l}
e_{\zeta_{1}}(t) \\
e_{\zeta_{2}}(t)
\end{array}\right]:=\left[\begin{array}{l}
\zeta_{1}(t)-x_{1, d}(t) \\
\zeta_{2}(t)-\dot{x}_{1, d}(t)
\end{array}\right]
$$
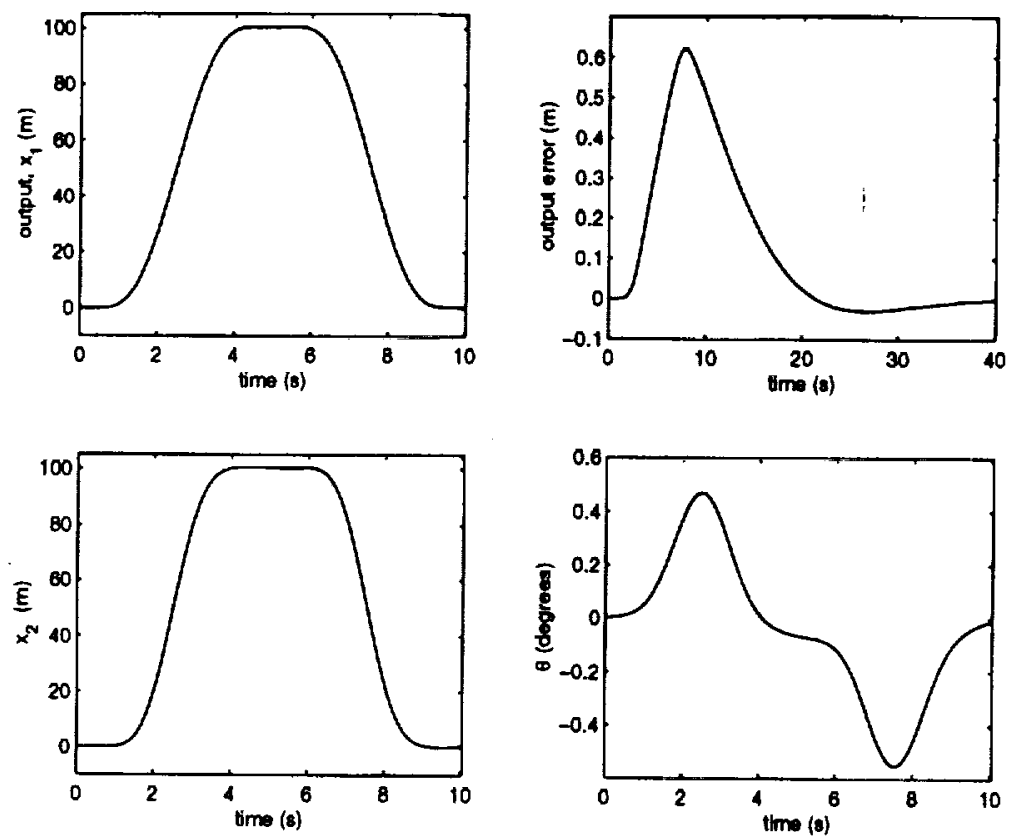

Figure 3. Results of Inversion

The additional input, $v$, is chosen $\left(v=F\left[\begin{array}{ll}e_{\zeta}^{T} & \eta^{T}\end{array}\right]^{T}\right)$ to remove the nonhyperbolicity. This can be achieved, for example, by pole-placement algorithms. Simulation results are presented next. The system parameters were chosen, for the simulation, as $K=10 \mathrm{~N} / \mathrm{m}, M=1 \mathrm{Kg}, l=9.8 \mathrm{~m}, g=9.8 \mathrm{~m} / \mathrm{s}^{2}$ and the $F$ used in simulations is

$$
F=\left[\begin{array}{llllll}
-4.52 e-2 & -3.04 e-1 & 5 e-4 & 1.5 e-3 & 0 & 0
\end{array}\right] \text {. }
$$

This $F$ removes the nonhyperbolicity of the internal dynamics and stable inversion of the modified inverse system is carried out using the algorithm in Section 3. Simulation results are presented in Figure 3, which shows the error caused in output-tracking due to redefinition of the output trajectory. The tracking error is less than $1 \%$ of the maximum value of the desired output (see Figure 3) - the rate at which the error goes to zero depends on the choice of $F$. The internal dynamics $\left(x_{2}, \theta\right)$ is also shown in Figure 3. Note that the nonhyperbolicity is circumvented and stable inversion is achieved with a relatively minor modification of the output.

\section{Conclusion}

A technique to achieve output tracking for nonminimum phase nonlinear systems with nonhyperbolic internal dynamics was presented. The approach integrated stable inversion techniques (that achieve exact tracking) with approximation approaches (that modify the internal dynamics) to remove the nonhyperbolicity of the internal dynamics. It was shown that, by giving up some of the precision in tracking, it is possible to achieve stable inversion of nonlinear nonminimum phase systems with nonhyperbolic internal dynamics.

\section{References}

[1] A. Isidori. Nonlinear Control Systems: An Introduction. Springler-Verlag, 1989.

[2] L. Qui and E. J. Davison. Performance limitations of non-minimum phase systems in the servomechanism problem. Automatica, 29, March:337-349, 1993. 
[3] S. Devasia, D. Chen, and B. Paden. Nonlinear inversion-based output tracking. IEEE Transactions on Automatic Control, 41(7):930-943, 1996.

[4] G. Meyer, L. R. Hunt, and R. Su. Nonlinear system guidance in the presense of transmission zero dynamics. NASA Technical Memorandun No. 4661, January, 1995.

[5] S. Devasia. Output tracking with non-hyperbolic and near non-hyperbolic internal dynamics: Helicopter hover control. AIAA J. of Guidance, Control, and Dynamics, 20(3):573-580, 1997.

[6] B.A. Francis and W. M. Wonham. The internal model principle of control theory. Automatica, 12(5):457$465,1976$.

[7] A. Isidori and C. I. Byrnes. Output regulation of nonlinear systems. IEEE Transactions on Automatic Control, 35(2):131-140, 1990.

[8] J. Huang. Output regulation of nonlinear systems with nonhyperbolic zero dynamics. IEEE Transactions on Automatic Control, 40(8):1497-1500, 1995.

[9] J. Huang and W. J. Rugh. An approximation method for the nonlinear servomechanism problem. IEEE Transactions on Automatic Control, Sept,37(9):1395-1398, 1992.

[10] A. J. Krener. The Construction of Optimal Linear and Nonlinear Regulators. In Systems, Models and Feedback: Theory and Application. A. Isidori and T.J. Tarn (editors). Birkhauser, Boston, 1992.

[11] S. Devasia, B. Paden, and C. Rossi. Exact-output tracking theory for systems with parameter jumps. International Journal of Control, 67(1):117-131, 1997.

[12] L. M. Silverman. Inversion of multivariable linear systems. IEEE Transactions on Automatic Control, 14(3):270-276, 1969.

[13] R. M. Hirschorn. Invertibility of multivariable nonlinear control system. IEEE Transactions on Automatic Control, 24(6):855-865, 1979.

[14] M. D. Di Benedetto and P. Lucibello. Inversion of nonlinear time-varying systems. IEEE Trans. Automatic Control, 38(8):1259-1264, 1993.

[15] S. Devasia and B. Paden. Exact output tracking for nonlinear time-varying systems. IEEE Transactions on Automatic Control, Feb, 43(2):283-288, 1998.

[16] B. Paden, D. Chen, R. Ledesma, and E. Bayo. Exponentially stable tracking control for multi-joint flexible manipulators. ASME Journal of Dynamic Systems, Measurement and Control, 115(1):53-59, 1993.

[17] S. Devasia and E. Bayo. Redundant actuators to achieve minimal vibration trajectory tracking of flexible multibodies: Theory and application. Journal of Nonlinear Dynamics, 6(4):419-431, 1994.

[18] P. Martin, S. Devasia, and B. Paden. A different look at output tracking: Control of a VTOL aircraft. Automatica, 32(1):101-107, 1996.

[19] R. Gurumoorthy and S. R. Sanders. Controling non-minimum phase nonlinear systems - the inverted pendulum on a cart example. Proceedings of the American Control Conference, San Francisco, CA, pages $680-685,1993$.

[20] Z. Xu and J. Hauser. Higher order approximate feedback linearization about a manifold for multi-input systems. IEEE Transactions on Automatic Control, 40(5):833-840, 1995.

[21] S. Gopalswamy and J.K. Hedrick. Tracking nonlinear non-minimum phase systems using sliding control. International Journal of Control, 57(5):1141-1158, 1993.

[22] Hassan K. Khalil. Nonlinear Systems. Macmillan, 1991.

[23] G. Meyer, L.R. Hunt, and R. Su. Nonlinear system guidance. Proceedings of the IEEE Conference on Decision and Control, New Orleans, Louisiana, pages 590-595, Dec 13-15, 1995.

[24] Jack K. Hale. Ordinary Differential Equations. Wiley-Interscience, 1969.

[25] Herbert Amann. Ordinary Differential Equations - An introduction to Nonlinear Analysis. Walter de Gruyter, New York, 1990. 